Proceedings of the 17th Czech and Slovak Conference on Magnetism, Košice, Slovakia, June 3-7, 2019

\title{
Domain Wall Dynamics of Wires in Perpendicular Magnetic Field
}

\author{
L. Fecova ${ }^{a, b, *}$, K. Richter ${ }^{a, c}$ AND R. VARGA ${ }^{b}$ \\ ${ }^{a}$ Institute of Physics, P.J. Safarik University, Park Angelinum 9, 04154 Kosice, Slovakia \\ ${ }^{b}$ CP M-TIP, P.J. Safarik University, Park Angelinum 9, 04154 Kosice, Slovakia \\ ${ }^{c}$ Institute of Materials Science, Kiel University, Kaiserstraße 2, 24143 Kiel, Germany
}

\begin{abstract}
The paper deals with the influence of an external perpendicular magnetic field on a domain wall motion in glass-coated amorphous microwires. The dependence of the domain wall velocity on the external axial magnetic field is measured at the presence of a perpendicular magnetic field. Subsequently, the microwire is rotated in a perpendicular field. The results show that the rotation of the microwire in the perpendicular magnetic field increases the domain wall velocity at certain rotations. At the same time, it shows that the mobility of the domain wall varies periodically with the rotation of a microwire. The results are discussed in terms of perpendicular magnetic anisotropy.
\end{abstract}

DOI: 10.12693/APhysPolA.137.849

PACS/topics: microwire, domain wall motion, perpendicular magnetic field

\section{Introduction}

Thin magnetic wires are of great interest for potential spintronic devices, in which domain wall motion is used to transfer information [1-3]. The velocity of the domain wall is a key factor that determines the operational functionality of modern spintronic devices. It has been shown that domain wall velocity can be tuned in several ways, like-electric current, the shape of the wire, or temperature. One of them is a perpendicular magnetic field, as well.

Amorphous glass-coated magnetic microwires are material characterized by fast domain wall motion. Due to the magnetoelastic interactions between the magnetic moments and the mechanical stresses induced during the production of the wires, their domain structure consists of one axial domain surrounded by a radial domain structure [4]. In addition, small closure domains are formed at the ends of the microwire to reduce the demagnetization fields. One of the most interesting properties of amorphous microwires is related to the observation of spontaneous magnetic bistability associated with a precisely rectangular hysteresis loop $[5,6]$.

Furthermore, their geometric and magnetic properties make them a great candidate for practical applications in spintronics and sensor technology $[7,8]$. Their use in practice [9] seems very promising especially because of their unique shape, size, biocompatibility, easy production, magnetic bistability, and the absence of the Walker limit $[5,10-12]$.

\footnotetext{
* corresponding author; e-mail

lucia.fecova@student.upjs.sk
}

Recently, even a relatively high domain wall velocity (up to $18 \mathrm{~km} / \mathrm{s}$ ) has been recorded, and it exceeds the estimated theoretical maximum of domain wall velocity $[5,6]$.

It has been shown that applying a positive perpendicular magnetic field and a negative magnetic field gives different velocity dependencies [13]. Such results were unexpected due to the cylindrical shape of the samples. Hence, our goal was to focus more on exploring the changing dynamics of the domain wall under the influence of the perpendicular magnetic field. Furthermore, we carried out a series of experiments in which we rotated microwire around its axis at the presence of a perpendicular magnetic field.

\section{Experimental}

Glass-coated amorphous microwire $\mathrm{Fe}_{76} \mathrm{Si}_{9} \mathrm{~B}_{10} \mathrm{P}_{5}$ (sample length $l_{v}=10 \mathrm{~cm}$, the diameter of metallic core $d=23 \mu \mathrm{m}$, the diameter of glass coat $D=59 \mu \mathrm{m}$ ) was used for measurements. In addition to the amorphous structure, the alloy is characterized by high saturation magnetization and low coercivity [14].

The Sixtus-Tonks method was used to investigate the domain wall dynamics of magnetic microwires [15]. This method involves a long primary coil with two sensing coils (with $4 \mathrm{~cm}$ distance in between). The primary coil generates a magnetic field (coil constant $k_{c}=12000 \mathrm{~m}^{-1}$ ) with frequency $f=1000 \mathrm{~Hz}$. The magnetic wire under investigation is placed inside the coil system so that when the domain wall of the microwire passes the sensing coils, an electromotive force is induced. The measured microwire was placed asymmetrically in the coil system, with one end placed inside the primary coil and second one the coil. Such a position of the microwire prevents multiple domain wall propagation [16]. 


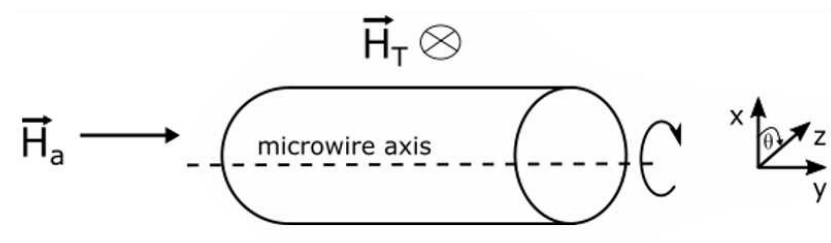

Fig. 1. A schematic image of a microwire rotated in a perpendicular magnetic field.

Since the whole measurement is very sensitive to external bias fields, the coil system with microwire was turned perpendicular to the direction of the Earth's magnetic field. Perpendicular magnetic field was applied by Helmholtz coils (coil constant $k_{c}=1560 \mathrm{~m}^{-1}$ supplied by the current source.

In addition, we have placed the entire coil system with the microwire into rotational sample holders. As a result, it was possible to rotate the microwire at $45^{\circ}$ (Fig. 1).

\section{Results and discussion}

At first, the domain wall velocity as a function of the axial magnetic is measured without an external perpendicular magnetic field (Fig. 2). The experiment shows that the dependence of the domain wall velocity from the external magnetic field is linear and increasing. It fulfils the relation:

$$
v=S\left(H-H_{0}\right),
$$

where $S$ is the mobility of the domain wall, and $H_{0}$ is the critical field. The domain wall mobility initially equals $S=22.8 \mathrm{~m}^{2} /(\mathrm{As}$ ) (Fig. 3). However, as can be seen in Fig. 3, the external magnetic field $H_{T}=55 \mathrm{~A} / \mathrm{m}$ applied perpendicularly, increases the value of the domain wall mobility to $S=38.8 \mathrm{~m}^{2} /(\mathrm{A} \mathrm{s})$.

Next, we measured domain wall velocity as a function of the axial magnetic field $H_{T}=55 \mathrm{~A} / \mathrm{m}$ in the presence of the perpendicular magnetic field when the microwire was rotated after $45^{\circ}$ angles around its axis (Fig. 2). We started at $0^{\circ}$, which was attributed to the current position of the microwire at the time of the first measurement. For any rotation of the microwire, the domain wall velocity increases linearly with perpendicular magnetic field (Fig. 2). The $45^{\circ}$ rotation of the microwire over the original position caused an even more significant change of the mobility (Fig. 3). Eventually, the domain wall mobility increased to $S=60.4 \mathrm{~m}^{2} /(\mathrm{A} \mathrm{s})$.

In addition, rotation after $45^{\circ}$ angles shows that there is a periodic change (increase and decrease) in mobility values (Fig. 3). Comparing the results of mobilities of the domain wall at individual angles of microwire rotation in the perpendicular magnetic field one can stated that the microwire is likely to be characterized by preferential directions corresponding to the rotations in which the domain wall moves faster after applying the perpendicular magnetic field.

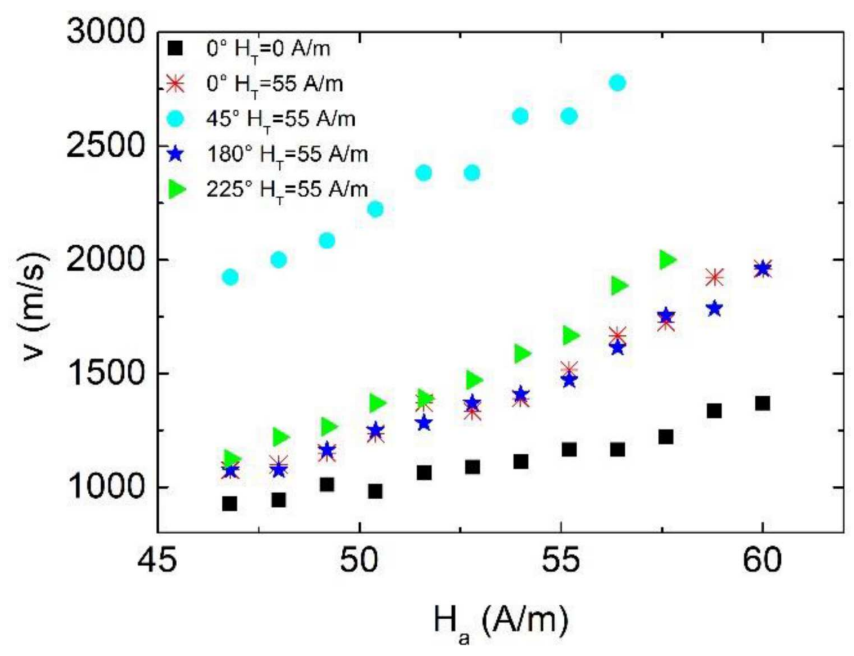

Fig. 2. The domain wall velocity as a function of the axial magnetic field, without a presence of the perpendicular magnetic field $\left(H_{T}=0 \mathrm{~A} / \mathrm{m}\right)$, and in the presence of the perpendicular magnetic field $\left(H_{T}=55 \mathrm{~A} / \mathrm{m}\right)$.

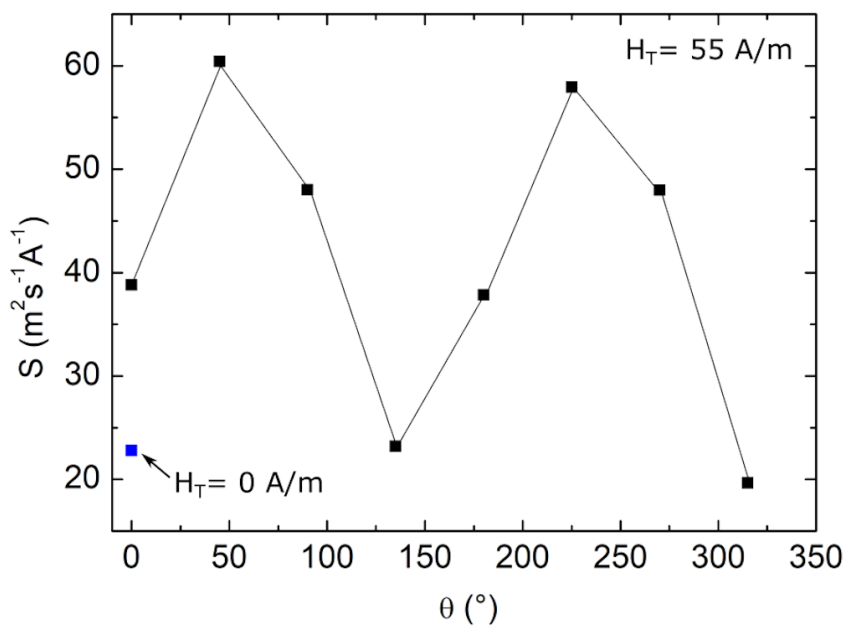

Fig. 3. Domain wall mobility as a function of the angle of the rotation in the perpendicular magnetic field $H_{T}$.

Due to the cylindrical shape of the microwire, observation of such an effect is, however, surprising. Traditionally, the radial symmetry of magnetization is expected in circular cross-section of microwire, so that rotation of the microwire in a perpendicular magnetic field would not caused different mobility values [11] Our results could be due to the presence of perpendicular magnetic anisotropy resulting from the production of the wires. During production by Taylor-Ulitovski method [17], the wires are cooled from one side by a water stream, resulting in a non-radial distribution of mechanical stresses (Fig. 4a, b). It can not be seen in amorphous wires but can be seen in wires with polycrystalline structure (Fig. 4a). 
(b)

$0^{\circ}$
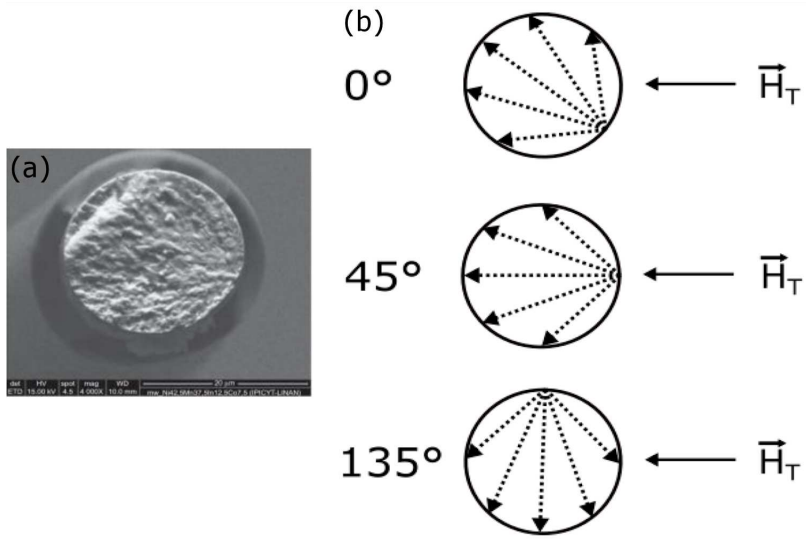

Fig. 4. (a) SEM picture of the cross-section of the $\mathrm{Ni}_{42.5} \mathrm{Mn}_{37.5} \operatorname{In}_{12.5} \mathrm{Co}_{7.5}$ microwire, and (b) anisotropy orientation with respect to the direction of the perpendicular magnetic field for three different angles of rotation.

When the direction of the perpendicular magnetic field is identical to the direction of the magnetic anisotropy, the domain wall width increases and its mobility is higher, as was the case with the $45^{\circ}$ angle of rotation. On the other hand, if the direction of the perpendicular magnetic field is perpendicular to the magnetic anisotropy, the domain wall width and its mobility decrease.

\section{Conclusions}

The domain wall dynamics of amorphous glass-coated microwire at the presence of the perpendicular magnetic field is studied in this work. It is shown that the domain wall velocity as a function of the external axial magnetic field remains linear and increasing at the presence of the perpendicular magnetic field, but its mobility varies considerably with certain angles of rotations as a result of perpendicular magnetic anisotropy.

\section{Acknowledgments}

This work was supported by the bilateral Slovak French project APVV SK-FR-2017-024, Slovak Grant Agency No. APVV-17-0184, APVV-16-0079, Slovak grant Agency VEGA 1/0195/18 and VEGA 1/0053/19.

\section{References}

[1] F. Ummelen, H. Swagten, B. Koopmans, Sci. Rep. 7, 833 (2017).

[2] L. O'Brien, A. Beguivin, D. Petit, A. FernandezPacheco, D. Read, R.P. Cowburn, Phil. Trans. R. Soc. A 370, 5794 (2012).

[3] C. Murapaka, P. Sethi, S. Goolaup, W.S. Lew, Sci. Rep. 6, 20130 (2016).

[4] N.N. Orlova, A.S. Aronin, S.I. Bozhko, Yu.P. Kabanov, V.S. Gornakov, J. Appl. Phys. 111, 073906 (2012).

[5] R. Varga, P. Klein, R. Sabol et al. in: High Performance Soft Magnetic Materials, Eds. A. Zhukov, Springer, 2017, p. 169.

[6] M. Ipatov, V. Zhukova, A.K. Zvezdin, A. Zhukov, J. Appl. Phys. 106, 103902 (2009).

[7] H. Pacaiova, M. Oravec, M. Šmelko, P. Lipovský, F. Forraj, J. Electr. Eng. 69, 493 (2018).

[8] K. Semrad, P. Lipovsky, J. Cernan, M. Jurcovic, Proc. Eng. 96, 428 (2014).

[9] M. Šmelko, M. Spodniak, K. Semrad, P. Tulipan, P. Lipovský, V. Moucha, in: 2018 XIII Int. Scientific Conference - New Trends in Aviation Development (NTAD), Kosice, 2018, p. 128.

[10] A.M. Aragón, M. Hernando-Rydings, A. Hernando, P. Marín, AIP Advances 5, 087132 (2015).

[11] M. Yan, A. Kákay, S. Gliga, R. Hertel, Phys. Rev. Lett. 104, 057201 (2010).

[12] R. Hertel, J. Magn. Magn. Mater. 249, 251 (2002).

[13] R. Varga, P. Klein, A. Jimenez, M. Vazquez, Phys. Stat. Sol. A. 2, 213 (2016).

[14] R. Varga, G. Infante, K. Richter, M. Vazquez, Phys. Stat. Sol. A. 3, 208 (2010).

[15] K.J. Sixtus, L. Tonks, Phys. Rev. 37, 8 (1931).

[16] J. Hudak, J. Blazek, A. Cverha, P. Gonda, R. Varga, Sensors Actuat. A Phys. 2, 156 (2009).

[17] S.A. Baranov, V.S. Larin, A.V. Torcunov, Crystals 7, 136 (2017). 